\title{
FLUID VELOCITY AND LIF TEMPERATURE MEASUREMENT IN A REAL MODEL OF HEAT EXCHANGER
}

\author{
Darina Jašíková, Petr Švarc, Václav Dvořák, Václav Kopeckỹ•
}

\begin{abstract}
This article presents the results of temperature measurements in a real model of heat exchanger using LIF method. By the help of fluorescent dye Rhodamine $B$ that responsive on the temperature variation is visualized the rate and warming - up of the liquid above the heating pipe. The drift excited by heat convection is measured by PIV method. The results of temperature field measurements are compared with metering affected by temperature probe.
\end{abstract}

\section{INTRODUCTION}

Nowadays the effort of many scientific teams is oriented on energy savings. The research is focused on prospecting feasibility of energy restoration and novel methods of natural resources exploitation. The latest acknowledgments are applied not only in the industry area, but also for households' usage. A good example is the solar domestic hot water storage (SDHW).

The SDHW system consists of the solar heating system (TES) and thermal energy storage unit. Presently the accumulation of heat is in most cases performed by waterfilled storage tank. As a heat medium pure water or its mixture with other suitable chemicals such as anti-freezers can be used. To achieve better efficiency of saving heat energy unit is utilized with a better thermal stratification. However the useful stratification can be disrupted by fluxion of storage medium in charging and discharging reservoir. A different physical process (kinetic energy of water entering the storage tank, thermal conduction, and diffusion of water with the walls of storage tank etc.) also has the negative influence.

To avoid above mentioned disturbing phenomenon, stratification can be influenced by different inner construction added into the storage tank. The placing of the inner construction also affects the implemented heating of the medium. The limited space around the implementation of the heating leads to the local temperature increase. The medium makes the thermal stratification more effective. This facts show the purpose of the medium dynamics investigation close to the heating unit.

It is possible to perform the numerical simulation, but only experimental data may show practicability and reliability of the simulation. To investigate the medium behavior in storage by means of numerical simulation, the two-dimensional model was created physically identical to the tracked region.

The temperature changes of the medium inside the heat exchanger give us the most important characteristics of the process itself. The common temperature measurement techniques mostly provide information just in one point and they are mostly based on invasive measurement methods that interrupt the inner flows. Such a pointed

- Ing. Darina Jašíková, FMIIS, TUL, Studentská 2, 46117 Liberec, Czech Republic, email:darina.jasikova@tul.cz

Ing. Petr Švarc, FS, TUL, Studentská 2, 46117 Liberec, Czech Republic

Ing. Václav Dvořák, Ph.D, FS, TUL, Studentská 2, 46117 Liberec, Czech Republic

Prof. Ing. Václav Kopecký, CSc., FMIIS, TUL, Studentská 2, 46117 Liberec, Czech Republic

This is an Open Access article distributed under the terms of the Creative Commons Attribution License 2.0, which permits unrestricted use, distribution, and reproduction in any medium, provided the original work is properly cited. 
measurement can be linked to the matrix of probes, but still doesn't provide us complex information over the whole investigated area. Today we can use the non-invasive, simultaneous, very accurate measurement technique, which gives us detailed information about the flow variations in time.

The Planar Laser Induced Fluorescent technique (PLIF) (Distelhoff et al.,1997) has a lot of various markers mostly based on the reaction principles (Shinohara et al., 2004) for tracing the temperature field as well as concentration changes or $\mathrm{pH}$ values monitoring.[1 - 3] Here we used a classical Rhodamine $B$ to investigate the temperature field changes. The Rhodamine $B$ fluorescent dye can be excited by common green laser with wavelength of $532 \mathrm{~nm}$. The study of the flow and its motion brings us very important knowledge of the system behavior. The most suitable non destructive method - the multi-dimensional technique Particle Image Velocimetry (PIV) can be applied for the flow characteristics examination.

Discussed here is a simplified model containing one heating element and the investigation is focused on the area just above the heating pipe. We were monitoring the temperature increase until we reached the medium temperature peak. This information gives us the complex view of the heating process and enables us to make the stratification more effective. The achievement of the high drift velocities raises the heattransfer coefficient.

\section{EXPERIMENTAL}

\subsection{Principles of PLIF and Flow velocity field investigation}

Planar laser-induced fluorescence (PLIF) is a fluorescence based technique used to perform non-intrusive whole field temperature measurements in liquids. The measurement is based on a well defined relationship between the intensity of the fluorescence and the temperature, while other experimental parameters constant.

The fluorescence physically corresponds to an atomic de-excitation process with emission of a photon of lower energy than the one which caused the excitation. When the matter is enlightened it re-emits light at a longer wavelength $\lambda_{\mathrm{em}}$ than the excitation wavelength $\lambda_{e x}$. The captured fluorescence is filtered out to reduce the excitation light. For this purpose is used the shift between excitation and emission spectra. This is enabled by optical filters $(570 \mathrm{~nm})$ that let only the re-emitted light to enter the detector.

The fluorescence intensity varies with the local excitation energy inside the sampling volume, the temperature $T$ inside volume $V$ and the physical properties of the medium.

Rhodamine $B$ is the most frequently used dye for planar-LIF measurements in liquids. This dye is highly soluble in water and is very chemically stable for very large range of concentration levels. The quantum efficiency of Rhodamine is very high, so it can give us very good fluorescence signals. The spectral characteristics of the dye are close to the $\mathrm{Nd}$ :YAG lasers (the lasers with wavelength $532 \mathrm{~nm}$ ). This wavelength is close to the optimal excitation wavelength of the Rhodamine B. (Fig. 1) The Rhodamine B solution responds to the temperature changes with decreases of the emitted light intensity.

Here the concentration of Rhodamine $B$ water solution was $0.1 \times 10^{-4} \%$.

The system layout enables the temperature and flow velocity measurement synchronisation. For the flow velocity measurement we used the PIV system with double 
pulse Nd:YAG laser and digital CCD camera with a spatial resolution of $1280 \times 1024$ pixels, both produced by Dantec Dynamics.

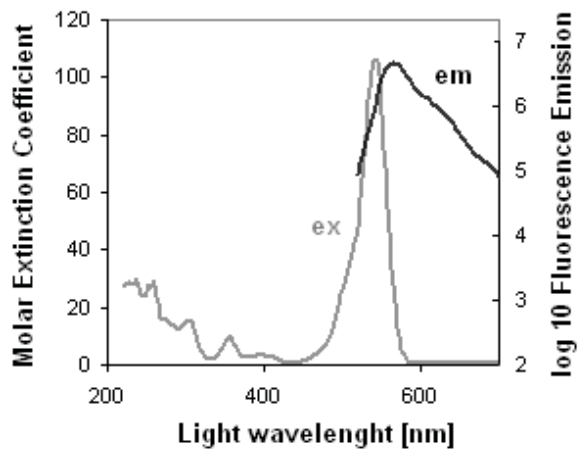

Fig. 1 Excitation and emittation light wavelengths of Rhodamine $B$.

\subsection{Heat exchanger configuration}

The simplified model containing just one heating element was built for the stratification examination. This testing unit was designed and manufactured from extruded acrylics (PMMA). The PMMA is suitable material for its physical and temperature properties. This model is one part of the complex heat exchanger; the proportions were kept in the ratio $1: 1$ to the real one. The model with the inner build and the heating pipe unit placement is shown on figure 2 a).
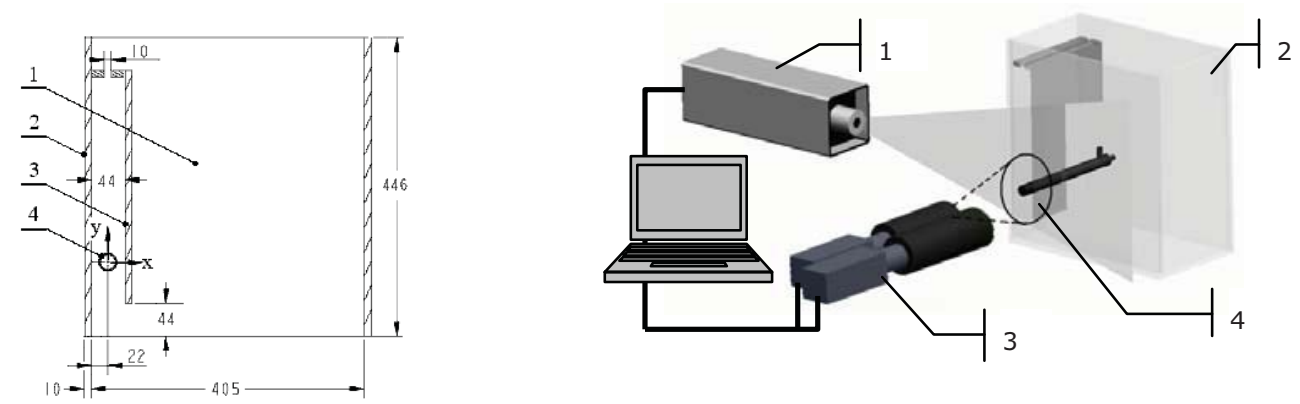

Fig. 2 a) The configuration and the proportions of the inner build. Setup of the heat exchanger model, where 1 is the medium (liquid with the physical properties of water), 2 the PMMA outer shell, 3 inner build and 4 is the heating pipe unit. b) The measurement setup for parallel investigation of temperature field and flow field variation in time. Under number 1 is the laser light source - Nd:YAG pulse laser with the light of wavelength 532 $\mathrm{nm}, 2$ is the heat exchanger with inner build unit, 3 the two cameras setup with special optical extension, 4 interrogation area - just above the heating pipe.

The model was included to the PLIF-PIV system layout. Both measurement techniques mentioned above can be combined into one experimental setup. Using this advanced 
alignment shown on figure 2b) we can get the results of temperature field and fluid velocities in one moment.

\section{Results}

The thermal induced flows and the performance of the heat exchanger is strongly influenced by the thermal stratification in the heat storage tank. There was found dependence between the performance and stratification [4-7]. The computer simulation relies on the accurate specification of the system's physical and thermal characteristics, and is often based on a number of simplifying assumptions. An important aspect of system performance, that can't be omitted, is storage heat loss characteristics.

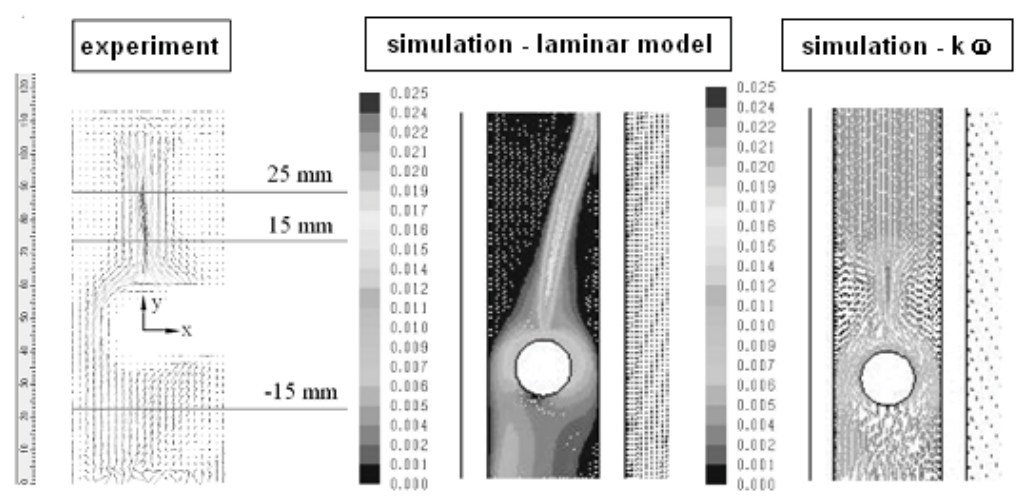

Fig. 3 Simulation calculated models and experimental results comparison with marked distance of analyzed vector profiles.

The designed heat exchanger was modelled using the Fluent 6.3 software. Computation region was represented with structured mesh in 2D Cartesian coordinates. The model geometry is coming out from real proportions of stratification placing.

The walls of the model are defined with physical properties of acrylic glass (PMMA) of constant density values $1180 \mathrm{~kg} \cdot \mathrm{m}^{-3}$, the heat conductivity $0.19 \mathrm{~W} .(\mathrm{m} . \mathrm{K})^{-1}$ and specific thermal capacity $1465 \mathrm{~J} \cdot(\mathrm{kg} \cdot \mathrm{K})^{-1}$. The model lid and the bilge are considered perfect heat isolated. The tank model is placed in the gravity field with gravitational acceleration

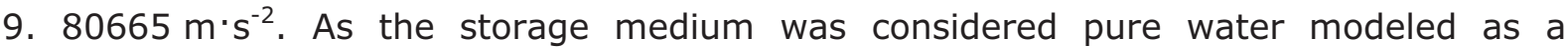
incompressible viscose liquid with constant heat conductivity $0.6 \mathrm{~W} \cdot(\mathrm{m} \cdot \mathrm{K})^{-1}$, specific thermal capacity $4182 \mathrm{~J} \cdot(\mathrm{kg} \cdot \mathrm{K})^{-1}$ and dynamic viscosity $1,003 \cdot 10^{-3} \mathrm{~Pa} \cdot \mathrm{s}$. The liquid density was defined as a polynomial regress function of absolute temperature used in temperature range $273.15 \mathrm{~K} \leq \mathrm{T} \leq 328.15 \mathrm{~K}$. The simulation of viscose flow was calculated in two different models.

The first model considered the dominant laminar viscosity, in second one was used two equation turbulence viscose model $k-\omega \mathrm{SST}$. For the calculation of this non stationary task was used the second order discrete schema in Fluent 6.3 software. Initialization of the calculation was performed for the medium temperature $22^{\circ} \mathrm{C}$. The surface of the heating pipe unit was as a boundary condition with constant value $47^{\circ} \mathrm{C}$. 
The experiment and the simulation proceeded in time steps 5 minutes during the 90 minutes measurement run. The maximal expected flow velocities were till $0.03 \mathrm{~m} \cdot \mathrm{s}^{-1}$. The investigation was focused on the area just above the heating pipe. We were monitoring the temperature evolution till we reached the medium temperature peak. This information gives us the complex view of the heating process and enables us make the stratification more effective. The raise of the drift velocities achieve the raise of the heat transfer coefficient. The 200 dataset were captured in every single time step for the statistical evaluation of flow velocity and stream behavior and also same value dataset of the temperature recording images. The experimental examination led to the calculation of absolute flow velocities vector maps, scalar velocity distribution and temperature field development in time. The results of the experimental measurement are show on Figure 4. The temperature records are in the good agreement with the flow field behavior.

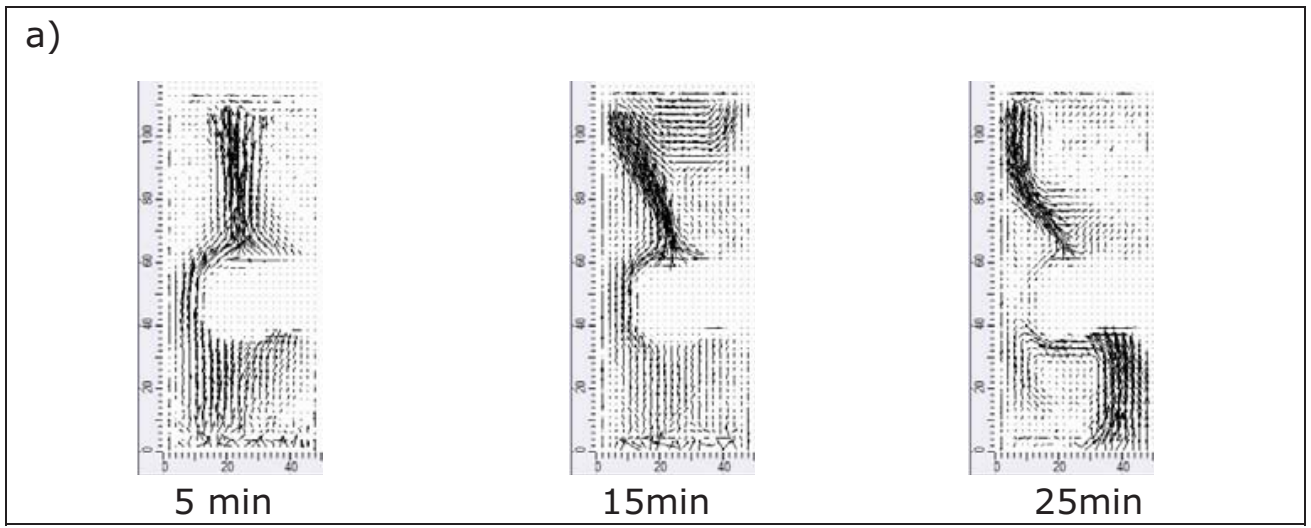

Fig. 4 a) The results of the experimental part. The table of pictures above shows the statistical vector map of calculated flow velocities in three selected time steps: 5,15 and 25 minutes

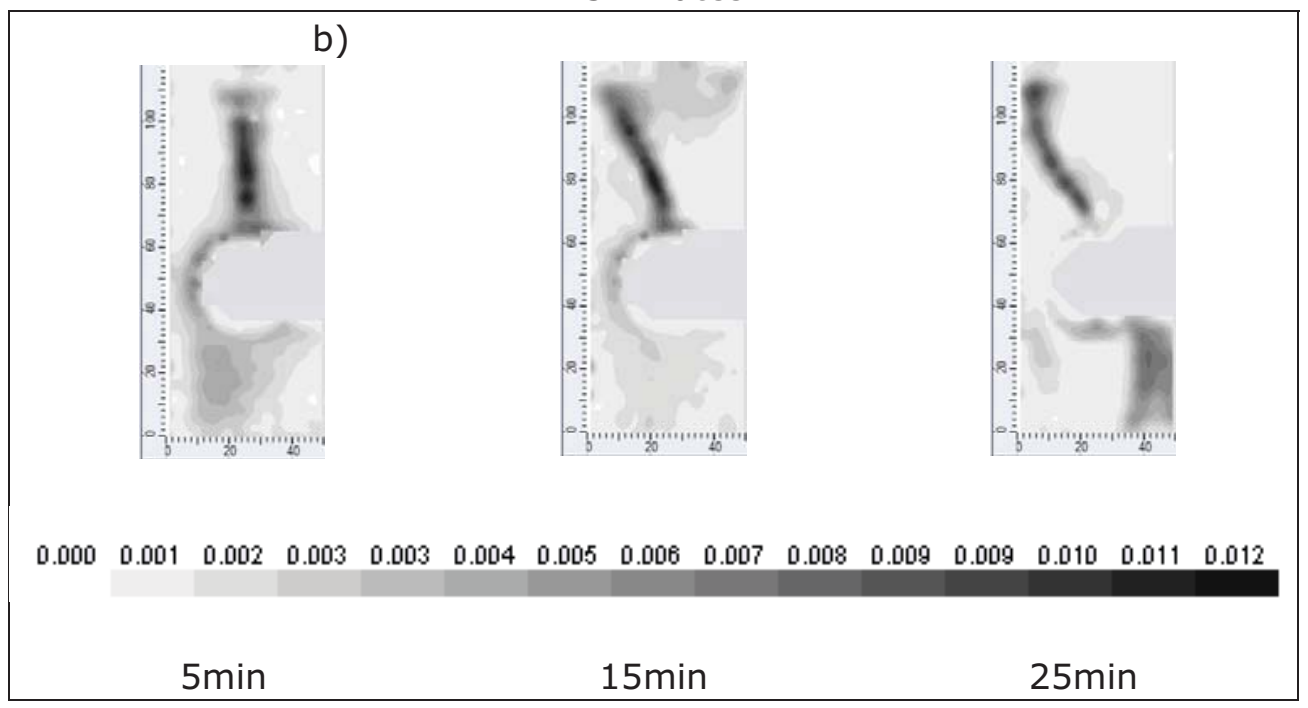

Fig. 4 b) The results of the experimental part. The scalar map of calculated flow velocities in three selected time steps: 5,15 and 25 minutes. 
c)

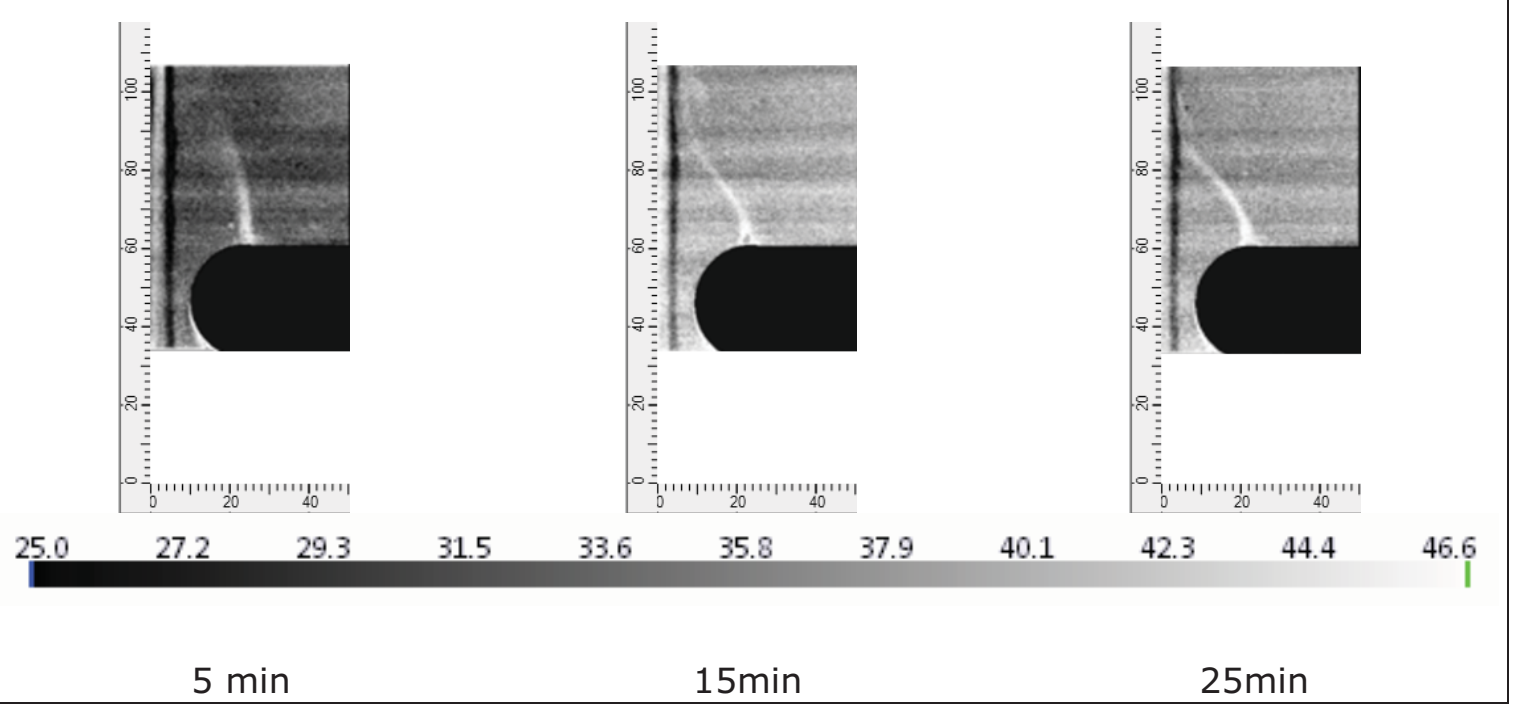

Fig. 4 c) The second part of the experimental datasets shows the results of temperature records.

The experimental datasets and their evaluated results were compared to the simulation outcomes. The comparison of the resulting datasets taken from experiments as well as simulation were taken in profiles $-15,15$ and $25 \mathrm{~mm}$ above and below from the surface of the heat pipe unit in the defined time steps. In these distances the profile plots were calculated and the charts are shown on figure 5.

From the quantitative point of view follows the evident disagreement between the velocities value from experimental and numerical calculations on every position. In the numerical models corresponds the values of flow velocities but they are qualitative different. In this case the laminar mode is in a very good agreement with the storage medium behavior and the experimental temperature charging. The difference in flow velocities could be caused by the mistake in the boundary conditions setting and the physical properties of the numerical calculation. The experimental setup investigated the area just above the heating pipe and didn't comprehend the full high of the tested equipment. In the room, where the heated medium conducts and the inner build in is narrowed, could be developed the vortex. The vortexes influence the liquid behavior in the whole area. 

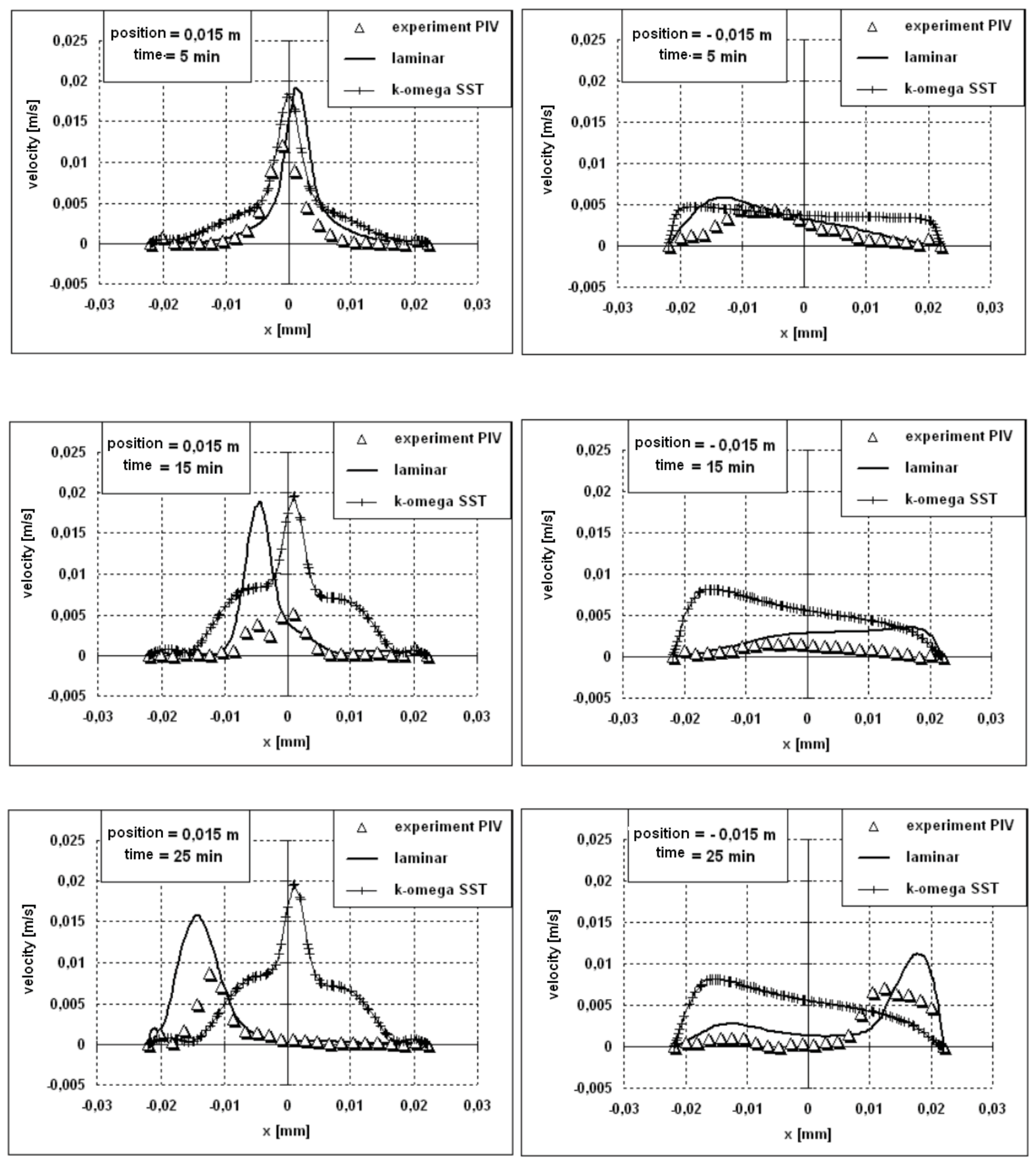

Fig. 5 The velocity profiles taken above and below the heating pipe unit in distance $15 \mathrm{~mm}$ from the pipe surface. The profiles develop in the time steps 5,15 and 25 minutes. 


\section{Discussion AND CONCLUSION}

The investigation of the effects formed during the storage medium heating up was realised using the visualisation of Particle Image Velocimetry technique parallel with Planar Laser Induced Fluorescence one. The experimental part of the research unveiled the disagreements in numerical simulation of the physical model. The measurements proved the suitability of the numerical model for needs of the more complex research. In the real model the fluid flow is more influenced by the complex inner arrangements of the heating up pipe system. The next research would follow the real model line in mapping the complex temperature and flow field characteristics

\section{Acknowledgement}

The project was provided with financial support by the MSM 4674788501 and GAAV 101/07/1499 foundation.

\section{REFERENCES}

[1] M.F.W. Distelhoff, A.J. Marquis, J.M. Nouri, J.H. Whitelaw: Scalar mixing measurements in batch operated stirred tanks, The Canadian Journal of Chemical Engineering 75, 1997, 641-652

[2] K. Shinohara, Y. Sugii, K. Okamoto, H. Madarame, A. Hibara, M. Tokeshi, T. Kitamori: Measurement of $\mathrm{pH}$ field of chemically reacting flow in microfluidic devices by laser-induced fluorescence. Measurement Science and Technology 15, 2004, 955-960

[3] S. Furbo, E. Andersen, at.al: Performance improvement by discharge from different levels in solar storage tanks, Solar Energy 79, 2005, 431-439

[4] M. Y. Haller, E. Yazdanshenas, at. al.: A method to determine stratification efficiency of thermal energy storage processes independently from storage heat losses, Solar Energy 84, 2010, 997-1007

[5] M. Y. Haller, C. A. Cruickshank, et. al.: Methods to determine stratification efficiency of thermal energy storage processes - Review and theoretical comparison, Solar Energy 83, 2009, 1847-1860

[6] E. Andersen, S. Furbo, J. Fan: Multilayer fabric stratification pipes for solar tanks, Solar Energy 81, 2007, 1219-1226

[7] C. A. Cruickshank, S. J. Harrison: Heat loss characteristics for a typical solar domestic hot water storage, Energy and Buildings 42, 2010, 1703-1710 